\title{
The Effect of Brain-computer Interface-based Cognitive Training in Patients with Dementia
}

\author{
Se-Jung Oh, PT, MS • Jeon-Nam Ryu, PT, $\mathrm{MS}^{\dagger}$
}

Department of Physical Therapy, Yeoju Institute of Technology

Received: August 21, 2018 / Revised: August 23, 2018 / Accepted: September 11, 2018

(c) 2018 J Korean Soc Phys Med

\section{| Abstract |}

PURPOSE: The purpose of the present study is to investigate the changes in the cognitive function of elderly dementia patients residing in a residential care facility, following six weeks of brain-computer interface (BCI)-based cognitive training and to determine whether BCI-based cognitive training effectively improves their cognitive functions.

METHODS: Thirty subjects diagnosed with dementia were randomly assigned to either the experimental or control group. Pre- and post-test cognitive function assessments were conducted using the mini mental state examination-Korean (MMSE-K) and Korean-dementia rating scale (K-DRS). The experimental group received BCI-based cognitive training, which consisted of games such as flying a ball and exploding a bomb, while the control group participated in music listening activities and National Health Gymnastics. Both groups engaged in a total of 18 sessions ( 3 times per week for

$\uparrow$ Corresponding Author : Jeon-Nam Ryu rjn8199@naver.com, http://orcid.org/0000-0002-6973-7225

This is an Open Access article distributed under the terms of the Creative Commons Attribution Non-Commercial License (http://creativecommons.org/licenses/by-nc/3.0) which permits unrestricted non-commercial use, distribution, and reproduction in any medium, provided the original work is properly cited.
6 weeks, for 40 minutes per session).

RESULTS: After 6 weeks of intervention, the experimental group had significantly increased MMSE-K scores $(19.53 \pm 1.30$ to $22.20 \pm 1.15 ; \mathrm{p}<.0011)$ and total K-DRS scores (87.20 \pm 4.16 to $99.33 \pm 1.15 ; \mathrm{p}<.0011)$. In addition, the experimental group showed greater cognitive improvements than the control group.

CONCLUSION: The results of this study suggest that BCI-based cognitive training is a positive intervention tool for improving the cognitive function of dementia patients.

Key Words: Brain-computer interface, Cognition, Dementia

\section{Introduction}

In Alzheimer's disease or vascular diseases, dementia is characterized by neurodegenerative changes that lead to cognitive impairment and cause emotional pain or distress to the patients and their families, as it restricts daily activities, reduces participation in social activities, and causes depression. Interventions that can restore the cognitive function and delay cognitive impairment have helped improve the quality of life and financial situation of these elderly patients (Zhu et al., 2013; Deary et al., 2013; Plassman et al., 2011).

Cognitive training involves a series of cognitive tasks usually related to memory, concentration, and problem 
solving. Cognitive training has been observed to increase brain activities associated with memory in elderly people with mild cognitive impairment (Belleville et al., 2011). Computerized cognitive training (CCT) has been shown to decelerate the loss of cognitive function in healthy elderly persons and those with cognitive impairments by activating cognitive function (Almeida et al., 2012). A recent review of the available literature reported that CCT improved the cognitive function of healthy elderly subjects and had some effect on the cognitive function and anxiety in patients with mild cognitive impairment; however, the effect size of CCT was small, and there were not enough high-quality studies in the review (Reijnders et al., 2013; García-Casal et al., 2017). CCT usually requires the help of a therapist or a caregiver and cannot be performed by healthy elderly individuals or those with cognitive impairment on their own.

The brain-computer interface $(\mathrm{BCI})$ refers to a technique in which the human brain is connected to a computer that analyzes the signals from cranial nerves in real time and transmits and communicates information without muscles or external devices (Broetz et al., 2010). The BCI has mainly been used to help quadriplegic patients or physically disabled patients by using real-time visual and auditory feedbacks (De Massari et al., 2013). Because active participation and practice are necessary to enable patients to use the $\mathrm{BCI}$, the use of $\mathrm{BCI}$ has been limited to patients who could fulfil this requirement. However, a study that used BCI-based games showed that elderly persons or patients with cognitive impairment could participate in cognitive training using the BCI (Liberati et al., 2012). Lee et al. (2013) reported that BCI-based game programs can improve the memory and attention span of health elderly persons. However, dementia patients with mild cognitive impairment who reside in residential care facilities, such as senior care facilities, have limited opportunities to participate in cognitive training and cannot perform cognitive training without help from a therapist.
The objective of this study was to investigate changes in the cognitive function of elderly dementia patients with mild cognitive impairment, residing in a residential care facility, following six weeks of BCI-based cognitive training and to determine whether BCI-based cognitive training effectively improved their cognitive functions.

\section{Methods}

\section{Participants}

Elderly persons aged 60 years or more who resided in senior care facilities, received long-term care insurance from the government, were diagnosed with Alzheimer's type dementia and vascular dementia were included. Thirty subjects who understood the content and purpose of this study signed the consent form (IRB No. MC13FISI0089). Patients with dementia who were unable to understand the content of the agreement received the consent of the family, and the rest of the subjects also received guidance and consent from their families.

Inclusion criteria were: 1) subjects who were diagnosed with dementia at least six months ago, 2) elderly persons who had MMSE scores of 18-23 points (Kramer et al., 1985), and 3) ability to sit independently on a chair for at least 30 minutes. Exclusion criteria were: 1) subjects who were undergoing other cognitive training, 2) previous history of epileptic seizure, and 3) visual or hearing impairment (Bae et al., 2012).

\section{Experimental procedures}

All subjects were subjected to pre-evaluation before study participation using the Korean-Mini-Mental Status Evaluation (MMSE-K) and the Korean version of Dementia Rating Scale (K-DRS). Subsequently, the 30 subjects were randomly divided into two groups using a number table. The experimental group was subjected to cognitive training using BCI-based games for six weeks, and the control group was subjected to music listening and National Health 
Gymnastics. All subjects participated in the training three times per week, 40 minutes per session, for six weeks. The subjects were re-evaluated after each training session.

The Mindset device (Mindset, Neurosky) was used during the BCI-based cognitive training. This device detects changes in brainwaves in the frontal lobe through a sensor attached to the subject's forehead and calculates the subject's concentration. The signals are converted to integers and sent to the computer via Bluetooth. A subject participating in the training sat comfortably on a chair using a headset and stared at a monitor screen $50 \mathrm{~cm}$ away. The subject played BCI-based cognitive training games that involved activities such as exploding bombs and floating balls (Kang et al., 2009). The bomb explosion game was played by a virtual avatar that burns objects (cars) from the hand and, with continued concentration, eventually explodes the objects. The bomb explosion game induced tension to activate the beta waves in charge of the conscious activities of the brain. The next game was the ball floating game. The ball floating game helped the subject focus his/her consciousness and reach a stable state to induce alpha waves. In this game, when the participant did not relax or concentrate on a floating ball, the ball slowly floated to the floor. The difficulty of the game gradually progressed from a light ball to a heavy ball. A bar graph and sounds were used to give real-time feedback on brain wave changes to help the subject focus on the training. The game difficulty was sequentially increased as the subject cleared each stage (Sung et al., 2012). To help the subjects understand how to participate in the training, simple feedback was provided during preliminary education and practice games. If subjects complained of tired eyes or fatigue, training was ceased and resumed after a sufficient break.

The control group participated in music listening activities and National Health Gymnastics according to the same schedule and for the same duration as the BCI training group. The titles and musical contents and how to perform the National Health Gymnastics were all explained to the subjects.

\section{Measurements and statistical analysis}

The MMSE was developed in 1975. The Korean version, developed in 1989, was used to evaluate the cognitive impairment of elderly subjects and identify those with dementia (Kwon and Park, 1989). The MMSE is a cognitive function assessment tool used to primarily identify and diagnose elderly persons who show clinical signs of cognitive impairment. The diagnostic reliability of this tool for dementia as a cognitive function assessment tool is .93 (Kim et al., 2003), and its reliability is .90 (Kim et al., 1998).

The K-DRS is the Korean version of the Dementia Rating Scale developed by Mattis that was refined and standardized, according to age and education level in Korea, to diagnose and evaluate the progress of Korean patients with dementia (Mattis, 1988). The K-DRS evaluates five domains of cognitive function that are important for diagnosing dementia. The domains include attention, the ability to receptively receive and process information and to concentrate; initiation and preservation, the ability to effectively execute an intended action; construction, spatiotemporal ability and motor reactions; conceptualization, the ability to abstractly process concrete concepts or events; and memory, the ability to remember, recall, or recognize information. The K-DRS consists of 36 tasks with a maximum total score of 144 . Higher scores indicate higher levels of cognitive function. The validity of this composition was .82 , and the reliability of evaluationreevaluation was .96 (Suk et al., 2010).

All measurements were analyzed using SPSS version 18.0 (SPSS Inc., Chicago, USA) for Windows. Descriptive statistics were used for the general characteristics of the subjects. An independent t-test was used to test the homogeneity of the variables. The Kolmogorov-Smirnov test was used to confirm the normality of the data. The 
Table 1. General characteristics of subjects

\begin{tabular}{lccc}
\hline & Control group $(\mathrm{n}=15)$ & Experimental group $(\mathrm{n}=15)$ & $\mathrm{p}$ \\
\hline Age (years) & $73.40 \pm 4.95$ & $71.00 \pm 3.63$ & .14 \\
Gender (male/female) & $6 / 9$ & $6 / 9$ & .39 \\
Education (years) & $2.60 \pm 2.61$ & $3.40 \pm 2.44$ & .36 \\
Hospitalization (month) & $2.33 \pm 1.05$ & $2.00 \pm .93$ & .40 \\
Duration of illness (years) & $2.93 \pm 1.33$ & $3.40 \pm 1.64$ & .68 \\
MMSE-K & $19.33 \pm 1.35$ & $19.53 \pm 1.30$ & .93 \\
K-DRS & $87.33 \pm 4.37$ & $87.20 \pm 4.16$ & \\
\hline
\end{tabular}

Values are presented as mean \pm standard deviation

MMSE-K : MiniMentalStateExamination-Koreanversion

K-DRS : Korean version of the Dementia Rating Scale

Table 2. The Comparison of MMSE-K Scores between and Within Two Groups

\begin{tabular}{cccc}
\hline & Control group $(\mathrm{n}=15)$ & Experimental group $(\mathrm{n}=15)$ & $\mathrm{p}$ \\
\hline Pre-test & $19.33 \pm 1.35$ & $19.53 \pm 1.30$ & $<.001^{* *}$ \\
Post-test & $19.00 \pm 1.31$ & $22.20 \pm 1.15$ & \\
$\mathrm{p}$ & .09 & $<.001^{*}$ & \\
\hline
\end{tabular}

MMSE-K : Mini Mental State Examination-Korean version

${ }^{*} \mathrm{p}<.05$ (within each group); ${ }^{* *} \mathrm{p}<.05$ (betweenexperimentalandcontrolgroups)

data were found to satisfy the normality. A paired t-test was performed to compare the values obtained before and after the experiment. An independent t-test was performed to test the homogeneity and the difference values between the two groups. The level of alpha was set at $\mathrm{p}<.05$.

\section{Results}

The general characteristics of the participants are shown in Table 1. The homogeneity test showed no significant difference between the two groups ( $>>.05)$.

The BCI-based cognitive training significantly increased the MMSE-K score from $19.53 \pm 1.30$ to $22.20 \pm 1.15$ ( $p<.05)$. The MMSE-K scores changed from $19.33 \pm 1.35$ to $19.00 \pm$ 1.31 in the control group; this change was insignificant. Comparison of the groups showed a greater increase in the BCI-based cognitive training group than in the control group $(\mathrm{p}<.05)($ Table 2.).
Comparison within the groups showed a significant increase in attention scores from $29.00 \pm .93$ points to $31.40 \pm 1.59$ in the BCI-based cognitive training group $(\mathrm{p}<.05)$. Initiation and preservation scores showed a statistically significant increase from $20.13 \pm 22.07$ to $22.07 \pm 1.62$ points, construction points showed a statistically significant increase from $3.60 \pm .51$ to $4.07 \pm .46$ points, conceptualization points showed a significant increase from $20.00 \pm 1.25$ to $22.27 \pm 1.87$ points, and memory points showed a significant increase from $14.47 \pm 1.77$ to $19.53 \pm 2.33$ points $(p<.05)$. Comparison between the two groups showed a more significant increase in all these scores in the BCI-based training group than in the control group $(\mathrm{p}<.05)($ Table 3.$)$.

\section{Discussion}

We investigated whether BCI-based cognitive training 
Table 3. The Comparison of K-DRS Scores between and Within Groups

\begin{tabular}{|c|c|c|c|c|c|c|c|c|}
\hline & \multicolumn{3}{|c|}{ Control group $(n=15)$} & \multicolumn{3}{|c|}{ Experimental group $(n=15)$} & \multirow{2}{*}{$\mathrm{t}$} & \multirow{2}{*}{$\mathrm{p}$} \\
\hline & Pre & Post & $\mathrm{p}$ & Pre & Post & $\mathrm{p}$ & & \\
\hline Total scores & $87.33 \pm 4.37$ & $86.87 \pm 3.89$ & .20 & $87.20 \pm 4.16$ & $99.33 \pm 3.70$ & $<.0011^{*}$ & 14.110 & $<.001 * *$ \\
\hline $\begin{array}{l}\text { Initiation and } \\
\text { Perseveration }\end{array}$ & $20.13 \pm 1.46$ & $20.13 \pm 1.46$ & 1.00 & $20.13 \pm 22.07$ & $22.07 \pm 1.62$ & $<.0011^{*}$ & -4.610 & $<.001^{* *}$ \\
\hline Construction & $3.47 \pm .52$ & $3.33 \pm .49$ & .16 & $3.60 \pm 0.51$ & $4.07 \pm .46$ & $.014 *$ & -2.824 & $<.001 * *$ \\
\hline Conceptualization & $19.73 \pm 1.28$ & $19.60 \pm 1.40$ & .16 & $20.00 \pm 1.25$ & $22.27 \pm 1.87$ & $<.0011^{*}$ & -4.900 & $<.001 * *$ \\
\hline Memory & $14.87 \pm 1.64$ & $14.73 \pm 1.22$ & .55 & $14.47 \pm 1.77$ & $19.53 \pm 2.33$ & $<.0011^{*}$ & -8.718 & $<.001 * *$ \\
\hline Attention & $29.13 \pm 1.13$ & $28.93 \pm .96$ & .27 & $29.00 \pm .93$ & $31.40 \pm 1.59$ & $<.0011 *$ & -5.392 & $<.001 * *$ \\
\hline
\end{tabular}

${ }^{*} \mathrm{p}<.05$ (within each group); ${ }^{* *} \mathrm{p}<.05$ (between experimental and control groups)

could effectively improve the cognitive function of elderly persons in senior care facilities. The cognitive training group who played BCI-based games for six weeks showed a more significant improvement in cognitive function than the group that listened to music and performed the National Health Gymnastics; therefore, the BCI-based cognitive training was found to be helpful for patients who suffer from dementia.

Cognitive function assessment and cognitive training for cognitive impairment that increases with age are important for dementia prevention. Existing CCT for patients with Alzheimer's disease (Cipriani et al., 2006; Jelcic et al., 2014; Lee et al., 2013) and mild cognitive impairment (Talassi et al., 2007) improved the MMSE scores and brain activities more effectively than other cognitive training programs and showed promising improvements in cognitive function. The individualized cognitive function assessments and the real-time feedback are strengths of CCT. Although CCT has been widely used in hospitals, minimal help from therapists is necessary for subjects who are not used to using computers. Both cognitive training with existing computers as well as BCI-based cognitive training have been shown to improve MMSE scores and high-level cognitive functions, such as attention improvement in dementia patients.

BCI-based cognitive training can be considered cognitive processing that mainly processes visual information and integrates previous experiences and sensory information from various domains of the central nervous system, like CCT (Park and Park, 2015). BCI-based training significantly improved memory, attention span, and the spatio-temporal ability of healthy elderly subjects. The dementia patients received feedback as they processed visual and auditory information during the BCI-based cognitive training and improved their visual perception through repeated training (Kim et al., 2013). In addition, we believe that the bomb explosion game in the BCI-based cognitive training caused tension to increase the subjects' conscious behaviors, and the ball floating game increased their attention in a relaxed state (Lee and Park, 2016). Therefore, the K-DRS results demonstrated that BCI-based cognitive training improved the subjects' attention skills, which allow the subjects to process information and focus on the training without being distracted. It also improved their ability to store, recall, and use memory.

Because of the small number of participants in the study, it is difficult to generalize the effect of the BCI-based cognitive training in dementia patients. Although the MMSE-K and K-DRS scores increased after six weeks of cognitive training using BCI-based games, the subjects were still classified as dementia patients who could have minimal cognitive impairment. Therefore, future studies 
should verify the effects of BCI-based cognitive training tools by including more subjects, analyzing the effects of these tools according to different levels of dementia, and investigating long-term and follow-up effects.

\section{Conclusion}

The dementia patients who participated in the BCI-based cognitive training showed significantly greater improvements in the MMSE-K and K-DRS scores compared with the control group who listened to music and performed the National Health Gymnastics. Like the existing CCT, the BCI-based cognitive training can be used as a basic tool to help improve the cognitive ability of dementia patients. Competitive advantages of the BCI-based cognitive training tools are its accessibility and ease of use compared to existing cognitive training tools. Therefore, BCI-based cognitive training may be used to prevent dementia among patients in care facilities who have less access to professional medical benefits.

\section{References}

Almeida OP, Yeap BB, Alfonso H, et al. Older men who use computers have lower risk of dementia. PLOS One. 2012;7(8):e44239.

Bae SJ, Jeong WS, Lee HG, et al. Effect of tDCS Stimulation for Improving Working Memory on Stroke Patients' EEG Variation. J korea Con Assoc. 2012;12(7): 261-72.

Belleville S, Clement F, Mellah S, et al. Training-related brain plasticity in subjects at risk of developing Alzheimer's disease. Brain. 2011;34(3):1623-34.

Broetz D, Braun C, Weber C, et al. Combination of braincomputer interface training and goal-directed physical therapy in chronic stroke: a case report. Neurorehabil Neural Repair. 2010;24(7):674-9.

Cipriani G, Bianchetti A, Trabucchi M. Outcomes of a computer-based cognitive rehabilitation program on Alzheimer"s disease patients compared with those on patients affected by mild cognitive impairment. Arch Gerontol Geriatr. 2006;43(3):327-35.

Deary IJ, Corley J, Gow AJ, et al. Age-associated cognitive decline. Br Med Bull. 2013;92(1):135-52.

De Massari D, Ruf CA, Furdea A, et al. Brain communication in the locked-in state. Brain. 2013;136(6):1989-2000.

Reijnders J, van Heugten C, van Boxtel M. Cognitive interventions in healthy older adults and people with mild cognitive impairment: a systematic review. Ageing Res Rev. 2013;12(1):263-75.

García-Casal JA, Loizeau A, Csipke E, et al. Computer-based cognitive interventions for people living with dementia: a systematic literature review and metaanalysis. Aging \& mental health. 2017;21(5):454-67.

Jelcic N, Agostini M, Meneghello F, et al. Feasibility and efficacy of cognitive telerehabilitation in early Alzheimer"s disease: A pilot study. Clin Interv Aging. 2014;9:1605-11.

Kang WK, Kang MH, Yang JL, et al. The comparison of EEG activity by computer assisted cognitive rehabilitation program in the normal elderly and the elderly with dementia. J Korean Soc Occup Ther. 2009;17(3):1-13.

Kim JM, Shin LS, Yoon JS, et al. Comparison of Diagnostic Validities between MMSE-K and K-MMSE for Screening of Dementia. J Korean Neuropsychiatr Assoc. 2003;42(1):124-30.

Kim MK, Lee SS, Lee YH, et al. An Investigation to the Diagnostic Validity of MMSE-K and the Psychopathology in a Group of Urban Patients with Dementia of Alzheimer Type. J Korean Neuropsychiatr Assoc. 1998;37(6):1277-90.

Kim SG, Kim CS, Kim K, et al. The Effect of EEG through Proprioceptive Exercise and Computerized Cognitive Therapy on Stroke. J Korean Soc Phys Med. 2013;8(4):505-12. 
Kramer M, German PS, Anthony JC, et al. Patterns of mental disorders among the elderly residents of eastern Baltimore. J Am Geriatr Soc. 1985;33(4):236-45. Kwon YC, Park JH. Standardization of Korean of the MiniMental State Examination (MMSE-K) for use in the elderly. J Korean Neuropsychiatr Assoc. 1989;28(1): 125-3.

Lee GY, Yip CC, Yu EC, et al. Evaluation of a computer assisted errorless learning-based memory training program for patients with early Alzheimer"s disease in Hong Kong: A pilot study. Clin Interv Aging. 2013;8:623-33.

Lee TS, Goh SJ, Quek SY, et al. A brain-computer interface based cognitive training system for healthy elderly: A randomized control pilot study for usability and preliminary efficacy. PLoS ONE. 2013;8(11):e79419.

Lee HS, Park YJ. The Effect of Physical Activity Program for Elderly with Dementia on Cognitive Function: Meta-Analysis of Studies in Korea. J Korean Soc Phys Med. 2016;11(2):115-21.

Liberati G, da Rocha JL, Van der Heiden L, et al. Toward a brain-computer interface for Alzheimer's disease patients by combining classical conditioning and brain state classification. J Alzheimers Dis. 2012;31(3): 211-20.
Mattis S. Dementia Rating Scale (DRS). Florida. Psychological Assessment Resources. 1988.

Park JH, Park JH. The effects of a Korean computer-based cognitive rehabilitation program on cognitive function and visual perception ability of patients with acute stroke. J Phys Ther Sci. 2015;27(8):2577-9.

Plassman BL, Langa KM, McCammon RJ, et al. Incidence of dementia and cognitive impairment, not dementia in the United States. Ann Neurol. 2011;70(3):418-26.

Suk JS, Chey JY, Kim HY. An Additional Normative Study of the Korean-Dementia Rating Scale. Kor J Clin Psychol. 2010;29(2):559-72.

Sung Y, Cho K, Um K. A development architecture for serious games using BCI (brain computer interface) sensors. Sensors (Basel). 2012;12(11):15671-88.

Talassi E, Guerreschi M, Feriani M, et al. Effectiveness of a cognitive rehabilitation program in mild dementia (MD) and mild cognitive impairment (MCI): A case control study. Arch Gerontol Geriatr. 2007;44:391-9.

Zhu CW, Sano M, Ferris SH, et al. Health-related resource use and costs in elderly adults with and without mild cognitive impairment. J Am Geriatr Soc. 2013; 61(3):396-402. 